\title{
Metaplasticity of Mossy Fiber Synaptic Transmission Involves Altered Release Probability
}

\author{
Ivan V. Goussakov, ${ }^{1}$ Klaus Fink, ${ }^{2}$ Christian E. Elger, ${ }^{1}$ and Heinz Beck ${ }^{1}$ \\ ${ }^{1}$ Department of Epileptology, University of Bonn, 53105 Bonn, Germany, and 2Department of Pharmacology, \\ University of Bonn, 53113 Bonn, Germany
}

Activity-dependent synaptic plasticity is a fundamental feature of CNS synapses. Intriguingly, the capacity of synapses to express plastic changes is itself subject to considerable activity-dependent variation, or metaplasticity. These forms of higher order plasticity are important because they may be crucial to maintain synapses within a dynamic functional range. In this study, we asked whether neuronal activity induced in vivo by application of kainate can induce lasting changes in mossy fiber short- and long-term plasticity.

Several weeks after kainate-induced status epilepticus, the mossy fiber, but not the associational-commissural pathway, exhibits a marked loss of paired-pulse facilitation, augmentation, and long-term potentiation (LTP). Because the adenylyl cyclase-protein kinase A cascade is involved in mossy fiber LTP induction, we have tested the integrity of this key pathway by pharmacological activation of either adenylyl cyclase or protein kinase A. These treatments resulted in LTP in control, but not in kainate-treated animals, indicating that statusinduced changes occur downstream of protein kinase A. To test whether altered neurotransmitter release might account for these changes, we measured the size of the releasable pool of glutamate in mossy fiber terminals. We find that the size of the releasable pool of glutamate was significantly increased in kainate-treated rats, indicating an increased release probability at the mossy fiber-CA3 synapse.

Therefore, we suggest that lasting changes in neurotransmitter release probability caused by neuronal activity may be a powerful mechanism for metaplasticity that modulates both short- and long-term plasticity in the mossy fiber-CA3 synapse after status epilepticus.

Key words: synaptic plasticity; paired-pulse facilitation; mossy fiber pathway; long-term potentiation; status epilepticus; release probability
The ability to modify synaptic strength in an activity-dependent manner, either as long-term depression (LTD) or long-term potentiation (LTP) is a fundamental feature of most CNS synapses. The properties of different forms of LTP in the rodent hippocampus have been exceedingly well studied. A less well studied but particularly intriguing finding is that the capacity of many synapses for plastic changes itself is subject to considerable activitydependent variation, or plasticity. This higher-order plasticity, or metaplasticity, may be important in normal function to keep synapses within a dynamic functional range, thus preventing them from entering states of saturated LTP or LTD (Abraham and Tate, 1997). In addition, such mechanisms may be invoked in diseases in which high-frequency discharges of hippocampal neurons occur, such as epilepsy. Metaplasticity has been observed experimentally as an inhibition of LTP or change in the frequency threshold between LTP and LTD by previous activation of NMDA receptors (Wang and Wagner, 1999). Conversely, a facilitation of LTP after metabotropic glutamate receptor activa-

\footnotetext{
Received Jan. 3, 2000; revised Feb. 14, 2000; accepted Feb. 17, 2000.

This work was supported by a grant from the Ministry of Science and Education, Nordrhein-Westfalen, a University of Bonn Medical Center grant "BONFOR", the Sonderforschungsbereich 400 of the Deutsche Forschungsgemeinschaft, the German-Israel collaborative research program of the Ministry of Science and the Bundesministerium fur Bildung, Wissenschaft, Forschung und Technologie, and the graduate program of the Deutsche Forschungsgemeinschaft "Pathogenese von Krankheiten des Nervensystems". We thank D. Langendörfer, H. Burisch, and M. Reitze for expert technical assistance.

Correspondence should be addressed to Dr. Heinz Beck, Department of Epileptology, University of Bonn Medical Center, Sigmund Freud Strasse 25, D-53105 Bonn, Germany. E-mail: heinz@mailer.meb.uni-bonn.de.

Copyright (C) 2000 Society for Neuroscience $\quad 0270-6474 / 00 / 203434-08 \$ 15.00 / 0$
}

tion has been described (Bortolotto et al., 1994; Cohen et al., 1998). These phenomena have been described at synapses in which LTP is dependent on the activation of NMDA receptors in the postsynaptic neuron, such as at the Schaffer collateral/ commissural-CA1 synapse.

A form of LTP distinct from that observed in the CA1 region can be observed at the mossy fiber-CA3 synapse. At this synapse, LTP induction seems to be independent of postsynaptic depolarization or $\mathrm{Ca}^{2+}$ influx through NMDA receptors (Zalutsky and Nicoll, 1990), but does seem to require an initial rise in postsynaptic $\mathrm{Ca}^{2+}$, engaging multiple mechanism other than NMDA receptors, i.e., via voltage-gated $\mathrm{Ca}^{2+}$ channels or intracellular $\mathrm{Ca}^{2+}$ release (Yeckel et al., 1999). In contrast to LTP induction, the expression or maintenance of fiber LTP seems to involve presynaptic changes in neurotransmitter release (Zalutsky and Nicoll, 1990; Nicoll et al., 1994; Xiang et al., 1994; Nicoll and Malenka, 1995). Genetic and pharmacological experiments have successfully uncovered some of the signal transduction pathways important in LTP expression, showing that both production of cAMP by the $\mathrm{Ca}^{2+}$-dependent type 1 adenylyl cyclase (Huang et al., 1994; Villacres et al., 1998) and activation of protein kinase A (Huang et al., 1995; Abel et al., 1997; Castillo et al., 1997) are necessary in the expression of mossy fiber LTP. In addition to unique features of LTP, the mossy fiber synapse characteristically exhibits paired-pulse facilitation to the second of two closely spaced stimuli. Paired-pulse facilitation or augmentation are most probably caused by a presynaptic accumulation of $\mathrm{Ca}^{2+}$ that leads to increased transmitter release (Regehr and Tank, 1991; Regehr et al., 1994; Salin et al., 1996). Thus, both short- and the 
expression of long-term plasticity at this synapse involve a change in neurotransmitter release.

In contrast to NMDA receptor-dependent LTP, it is not known whether mossy fiber LTP shows metaplasticity. We have therefore investigated short- and long-term plasticity at the mossy fiber synapse several weeks after status epilepticus induced by kainate application in vivo. Our data indicate that neuronal activity can cause a lasting change in short- and long-term plasticity via an upregulation of neurotransmitter release probability.

\section{MATERIALS AND METHODS}

Kainate treatment of rats. Adult male Sprague Dawley rats (30-31 d; 80-140 gm) were injected twice with kainic acid (13.0 mg/kg, i.p.) on 2 consecutive days. All animals were observed for a time period of 4-6 hr after injection. Kainate-treated rats showed an onset of seizure activity 15-20 min after kainic acid administration. Seizures were rated according to the scale of Racine (1972). Status epilepticus was defined as continuous limbic motor seizures of stage 2 or higher. Eighty percent of the animals showed status epilepticus with bouts of generalized tonicclonic seizures after the second injection lasting for 1-2 hr. Only these animals were used for further electrophysiological experiments. After a latent period ranging from 2-3 weeks, spontaneous seizures appeared in these animals. To exclude contamination by short-term seizureassociated effects, rats were killed only after an $8 \mathrm{hr}$ seizure-free period. Both rats injected with saline vehicle or age-matched control rats were used as a control group.

Preparation and recording configuration. Kainate-treated and control rats were decapitated under chloroform anesthesia. The brain was rapidly removed and transferred to ice-cold saline (in $\mathrm{mm}$ : $\mathrm{NaCl} 125.0, \mathrm{KCl} 3.0$, $\mathrm{CaCl}_{2} 2.5, \mathrm{MgCl}_{2} 1.3, \mathrm{Na}_{2} \mathrm{HPO}_{4} 1.25, \mathrm{NaHCO}_{3} 26.0$, and D-glucose 13.0, $\mathrm{pH}$ 7.4) bubbled with $95 \% \mathrm{O}_{2}$ and $5 \% \mathrm{CO}_{2}$. Four hundred micrometer coronal hippocampal slices were prepared with a vibratome (model 1000S; Leica, Wetzlar, Germany) and transferred to an interface chamber where they were continuously superfused with saline of the above composition $(1.8 \mathrm{ml} / \mathrm{min})$. The temperature of the recording chamber was maintained at $30^{\circ} \mathrm{C}$. Mossy fiber field potentials were recorded in the CA3 stratum lucidum. Care was taken to minimize the contribution of fibers other than mossy fibers to the field EPSPs (fEPSPs) (Claiborne et al., 1993; Castillo et al., 1996). First, the stimulation electrode was placed at a location in the granule cell layer of the dentate gyrus in which stimulation of the CA3 stratum lucidum produced the maximal antidromic field potentials. Second, the reversal of the waveform as the recording electrode was moved from the stratum lucidum to the stratum radiatum served to define the extent of the mossy fiber input in the CA3 region. Third, the extent of the stratum lucidum was delineated by moving the recording electrode in a direction perpendicular to the CA3 pyramidal cell layer until a reversal of mossy fiber fEPSPs could be observed. Positivities after the mossy fiber fEPSP were minimized because these may reflect contamination by disynaptic excitatory input. In this recording configuration, the application of the group II metabotropic glutamate receptor antagonist $\left(\left(2 S, 2^{\prime} R, 3^{\prime} R\right)-2-\left(2^{\prime}, 3^{\prime}\right.\right.$-dicarboxycyclopropyl) glycine $(1 \mu \mathrm{M})$ that selectively inhibits mossy fiber but not associationalcommissural EPSPs (Yeckel et al., 1999) blocked $86.1 \pm 5.6 \%$ of the fEPSP $(n=5)$. In addition, recordings in which the EPSP shape or latency changed markedly after LTP induction were excluded because such changes may also be caused by disynaptic input via associationalcommissural fibers (Claiborne et al., 1993). In all recordings of mossy fiber responses, $25 \mu \mathrm{M}$ D-APV was added to the saline to block NMDAmediated responses. The associational-commissural input into the CA3 region was stimulated by placing both the stimulation electrode and the recording electrode in the stratum radiatum and omitting D-APV from the bath solution. Stimulation was performed with two $0.1 \mathrm{msec}$ current pulses with an interstimulus interval of $50 \mathrm{msec}$ delivered via a bipolar platinum stimulation electrode at $0.05 \mathrm{~Hz}$. The baseline stimulation strength was adjusted to elicit a fEPSP of $\sim 60 \%$ of the maximal fEPSP amplitude. LTP in the mossy fiber as well as associational-commissural pathway was elicited with two consecutive tetani $(1 \mathrm{sec}, 100 \mathrm{~Hz}$ each, 10 sec apart) at twice the baseline stimulation intensity. In some experiments, potentiation was elicited by a transient $(20 \mathrm{~min})$ application of the adenylyl cyclase activator forskolin $(50 \mu \mathrm{M})$ applied together with the phosphodiesterase inhibitor 3-isobutyl-1-methylxanthine (IBMX; 50 $\mu \mathrm{M})$. In these experiments, $25 \mu \mathrm{M}$ APV was applied $10 \mathrm{~min}$ before and 40 min after the application of forskolin/IBMX. Field potentials were recorded with borosilicate glass microelectrodes $(\sim 2 \mathrm{M} \Omega)$ filled with artificial CSF. Signals were amplified with a field potential amplifier (Charité, Berlin, Germany), filtered at $3 \mathrm{kHz}$ and digitized with a sampling frequency of $10 \mathrm{kHz}$ (ITC-16; Instrutech, Minneola, FL). Data were then transferred to hard disk for off-line analysis with the TIDA for Windows 3.01 acquisition and analysis package (HEKA Elektronik, Lambrecht-Pfalz, Germany). Data were monitored on-line with an oscilloscope (Hameg, Frankfurt, Germany) and a chart recorder (Astro-Med, West Warwick, RI). Data are given as mean \pm SEM.

Preparation of synaptosomes. CA3 regions were dissected from the hippocampus of male Sprague Dawley rats (220-350 gm; Charles River, Sulzfeld, Germany) according to Hortnagl et al. (1991). Large mossy fiber synaptosomes were prepared as described before (Lonart and Südhof, 1998; Lonart et al., 1998). Isolated CA3 regions were manually homogenized in $1 \mathrm{~mm} \mathrm{MgSO}_{4}, 0.3 \mathrm{M}$ sucrose, and $15 \mathrm{~mm}$ HEPES at $\mathrm{pH}$ 7.4 on ice and centrifuged for $10 \mathrm{~min}$ at $900 \times g$. The pellet containing large synaptosomes and nuclei was resuspended in $18 \%$ ficoll and $0.3 \mathrm{M}$ sucrose and centrifuged $20 \mathrm{~min}$ at $16,000 \times g$. The supernatant containing the large synaptosomes was diluted 10 times with $0.3 \mathrm{~m}$ sucrose and centrifuged $20 \mathrm{~min}$ at $15,000 \times \mathrm{g}$. The resulting pellet was resuspended in modified Krebs' buffer composed of (in $\mathrm{mm}$ ): $\mathrm{NaCl} 118, \mathrm{KCl} 4.8$, $\mathrm{NaHCO}_{3} 25, \mathrm{KH}_{2} \mathrm{PO}_{4} 1.2, \mathrm{CaCl}_{2} 1.3, \mathrm{MgSO}_{4} 1.2$, D-glucose 11.1, ascorbic acid 0.06, and disodium EDTA 0.03 (equilibrated with $95 \% \mathrm{O}_{2}$ and $5 \% \mathrm{CO}_{2}, \mathrm{pH} 7.4,4^{\circ} \mathrm{C}$ ) and kept on ice for $90 \mathrm{~min}$.

Measurement of ${ }^{3} \mathrm{H}$-glutamate release. To label the readily releasable glutamate pool, synaptosomes were incubated with $200 \mathrm{nM} \mathrm{L}-\left[3,4-{ }^{3} \mathrm{H}\right]-$ glutamic acid (specific activity $44 \mathrm{Ci} / \mathrm{mmol}$; NEN, Dreieich, Germany) for $5 \mathrm{~min}$ at $35^{\circ} \mathrm{C}$. Superfusion was performed as described before (Fink and Göthert, 1992) with modifications. Aliquots of the labeled synaptosomal suspension (final protein content, $363 \pm 49 \mu \mathrm{g} / \mathrm{ml}$ ) were layered on Whatman (Maidstone, UK) GF/B filters in chambers and superfused with Krebs' buffer at $33^{\circ} \mathrm{C}$ with a flow rate of $0.8 \mathrm{ml} / \mathrm{min}$ continuously equilibrated with $95 \% \mathrm{O}_{2}$ and $5 \% \mathrm{CO}_{2}$. Ten minutes after the start of superfusion, tritium overflow was stimulated by addition of $0.5 \mathrm{M}$ sucrose to the superfusion buffer for $30 \mathrm{sec}$ administered by a rapidly switching programmable buffer supply system. The superfusate was continuously collected in $1 \mathrm{~min}$ fractions. Radioactivity of superfusion fractions and synaptosmes was determined by liquid scintillation counting (Beckman 1801; Beckman Instruments, Fullerton, CA). Tritium efflux was calculated as the fraction of tritium content in the synaptosomes at the beginning of the respective collection period. Basal tritium efflux was assumed to be stable or decline linearly during the fraction collection period. Sucrose stimulation-evoked tritium overflow was calculated by subtracting basal efflux from total overflow and considered to reflect ${ }^{3} \mathrm{H}$-glutamate release. In $\mathrm{Ca}^{2+}$-free experiments, $\mathrm{CaCl}_{2}$ was omitted from the buffer throughout superfusion. Data are given as means \pm SEM from five or six experiments, each done five to eight times. For statistical evaluation, a two-tailed paired Student's $t$ test was used, with a significance level of $p<0.05$.

\section{RESULTS \\ Properties of mossy fiber fEPSPs in the kainate-treated animals}

Mossy fiber fEPSPs were elicited with a stimulation electrode placed adjacent to the granule cell layer and a recording electrode placed in the CA3-stratum lucidum (Fig. 1 $A$ ). fEPSP amplitudes increased with stimulation strength in both the kainate and the control group (Fig. 1 $A$ ). Input-output curves were constructed from the average normalized fEPSP amplitudes plotted versus the stimulation intensity (Fig. 1B). The input-output curve of the kainate group $(n=11)$ was shifted toward lower stimulation strengths compared to control animals $(n=8)$. The maximal amplitude of potentials in the kainate group was not significantly different from those measured in the control group $(2.8 \pm 1.6$ and $2.6 \pm 1.3 \mathrm{mV})$.

\section{Paired-pulse facilitation and augmentation}

Paired-pulse facilitation is a form of short-term plasticity that provides a measure of the ability of a chemical synapse to increase transmitter release in response to the second of two closely spaced action potentials. It is thought that this increased trans- 

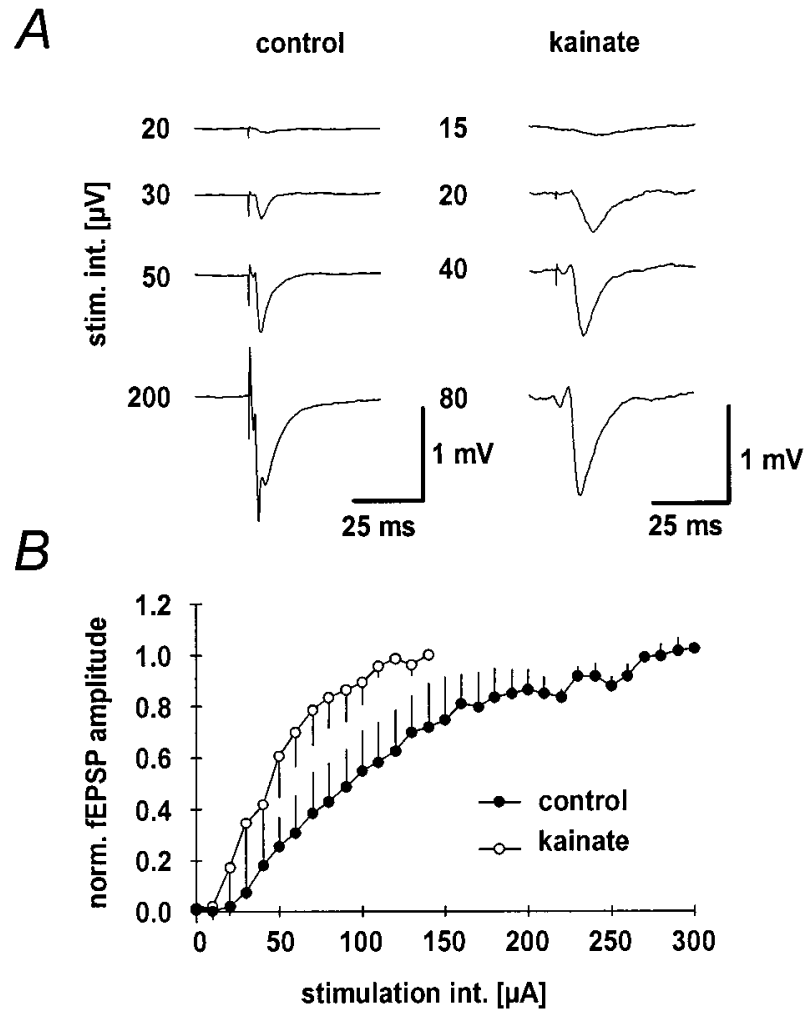

Figure 1. Mossy fiber fEPSPs in kainate-treated and control rats. $A$, Representative fEPSPs at different stimulation intensities for control (left panel) and kainate-treated (right panel) animals. $B$, Input-output curves constructed from the normalized fEPSP peak amplitudes for kainatetreated $(n=11$; open symbols $)$ and control $(n=8$; filled symbols $)$ rats.

mitter release is caused by an intraterminal $\mathrm{Ca}^{2+}$ accumulation that enhances $\mathrm{Ca}^{2+}$-dependent neurotransmitter release (Regehr and Tank, 1991; Regehr et al., 1994). When paired-pulse stimulation with an interpulse interval of $50 \mathrm{msec}$ was performed, kainate-treated animals showed markedly less paired-pulse facilitation (kainate-treated animals: $1.91 \pm 0.25, n=9$; control animals: $1.36 \pm 0.24, n=11$; Fig. $2 A 1)$. This finding was stable over a wide range of stimulation intensities (Fig. 2A2). Similarly, pronounced augmentation was observed in control animals when the mossy fiber-CA3 pathway was stimulated with a stimulus train at $100 \mathrm{~Hz}$, whereas kainate-treated animals showed no augmentation ( $n=3$ and $n=5$, respectively; Fig. $2 B$ ).

\section{Tetanus-induced changes in synaptic efficacy and paired-pulse facilitation}

Next, we have examined the changes in fEPSP slope and pairedpulse facilitation after a high-frequency tetanus applied to the mossy fiber pathway in both control and kainate-treated animals. As expected, after tetanization of the mossy fiber pathway, the fEPSP slope showed significant potentiation in control animals (Fig. 3A1, A2, filled symbols). Mossy fiber LTP in control animals was accompanied by a reduction in paired-pulse facilitation (Fig. 3B1, B2, filled symbols). This effect was transient, and pairedpulse facilitation decayed to baseline levels within $30 \mathrm{~min}$ (Fig. $3 B 1$, filled symbols). In marked contrast to control animals, a high-frequency tetanus applied to the mossy fiber pathway resulted in significantly less posttetanic potentiation in kainatetreated animals. In addition, the fEPSP slope decayed to baseline within 40 min after tetanus in kainate-treated animals (Fig. 3A1,
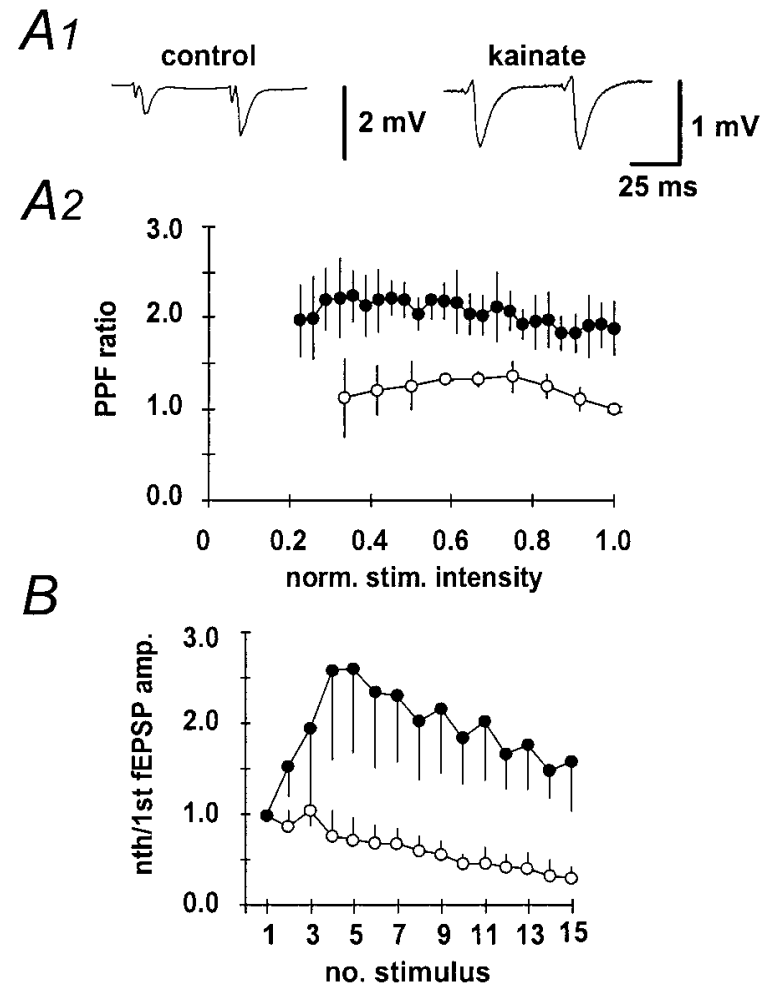

Figure 2. Paired-pulse facilitation in the mossy fiber pathway in kainatetreated and control rats. $A 1$, Representative fEPSPs to paired-pulse stimulation with an interpulse interval of $50 \mathrm{msec}$. $A 2$, The amplitude ratio of second to first fEPSP at different stimulation intensities in the kainate $(n=6$; open symbols) and control $(n=6$; filled symbols) groups. $B$, fEPSP amplitudes to continuous high-frequency stimulation of the mossy fiber pathway $(100 \mathrm{~Hz}, 15$ impulses), normalized to the amplitude of the first fEPSP in the kainate $(n=3$; open symbols $)$ and control $(n=$ 5; filled symbols) groups.

A2, open symbols). Consistent with this lack of LTP, no changes in paired-pulse facilitation could be observed in kainate-treated rats after tetanic stimulation (Fig. 3B1,B2, open symbols). As already suggested by the parallel changes in paired-pulse facilitation, LTP, and posttetanic potentiation, these parameters were correlated in control animals $(p<0.01)$. Thus, in this group the amount of paired-pulse facilitation or posttetanic potentiation is predictive of the amount of LTP that can be induced at the mossy fiber-CA3 synapse [Fig. 4A1, A2, open symbols (kainate-treated animals), filled symbols, (control animals)].

The changes in paired-pulse facilitation and LTP were specific to the mossy fiber pathway, because LTP induced at the associational-commissural pathway in the absence of DAPV was not different in kainate-treated $(135 \pm 22 \% ; n=6)$ compared to control animals (126 $\pm 11 \%$ of baseline fEPSP slope; $n=4$; Fig. $5 A 1, A 2)$. Thus, the observed changes in short- and long-term plasticity seemed to be confined to the mossy fiber-CA3 synapse.

\section{Induction of potentiation by pharmacological activation of adenylyl cyclase or protein kinase $\mathrm{A}$}

Next, we have directly activated key enzymes important in the induction of mossy fiber LTP. A large body of evidence suggests that the adenylyl cyclase-protein kinase A pathway is important in the expression of mossy fiber LTP (Huang et al., 1994; Weisskopf et al., 1994; Huang et al., 1995; Abel et al., 1997; Villacres et al., 1998). First, we have therefore directly raised the intraterminal cAMP concentration by applying the adenylyl cyclase activator 

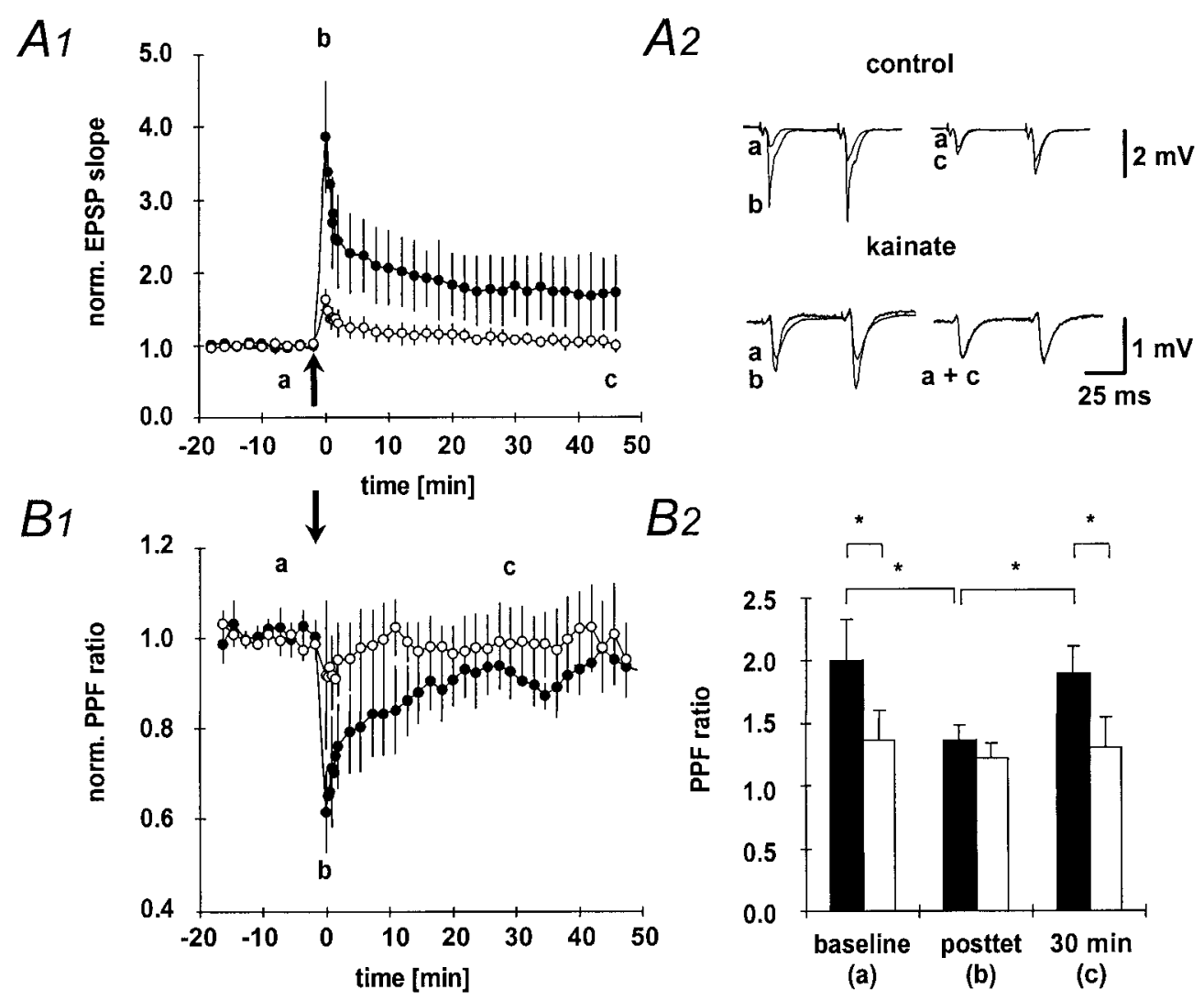

forskolin $(50 \mu \mathrm{M})$ together with the phosphodiesterase inhibitor IBMX (50 $\mu \mathrm{M}$; Fig. 6A2, horizontal bar) (Huang et al., 1994; Weisskopf et al., 1994). This manipulation circumvents the steps preceding activation of adenylyl cyclase otherwise necessary in LTP induction. Application of forskolin/IBMX led to a pronounced potentiation of the fEPSP slope in control animals and a much smaller potentiation in kainate-treated animals within $20-25 \min (486 \pm 118$ and $334 \pm 58 \%$ of baseline fEPSP slope, respectively; Fig. $6 A 1, A 2)$. In the kainate group, the potentiation decayed to baseline levels $(95 \pm 21 \% ; n=6)$ within $2 \mathrm{hr}$ (Fig. $6 A 1$, right panel, $A 2$, open symbols). In control animals, the potentiation showed a very slow decay before stabilizing $3 \mathrm{hr}$ after washout of forskolin/IBMX at $188 \pm 58 \%$ of the baseline fEPSP slope ( $n=8$; Fig. 6 A1, left panel, A2, filled symbols). Similar to LTP, the paired-pulse facilitation was not altered significantly in the kainate-treated rats by application of forskolin/IBMX, although a prominent reduction in paired-pulse facilitation could be observed in control animals (data not shown). As described previously (Huang et al., 1994), pharmacologically induced potentiation largely occluded subsequent LTP induced by tetanic stimulation $(100 \mathrm{~Hz}, 1 \mathrm{sec}$; Fig. 6A2).

Next, we have performed a similar experiment using the protein kinase A agonist spcAMPs. After an initial depression probably caused by activation of adenosine receptors (Frey et al., 1993; Huang et al., 1994; Weisskopf et al., 1994), activation of protein kinase A led to a slow increase in the fEPSP slope that persisted for up to $4 \mathrm{hr}$ in control animals (260 $\pm 104 \%$ of baseline fEPSP slope; $n=6$; Fig. 7A1,A2, filled symbols). No change in fEPSP slope could be observed in kainate-treated animals $(105 \pm 20 \%$ of baseline fEPSP slope; $n=5$; Fig. 7A1,A2, open symbols). These experiments suggest to us that signal transduction elements downstream from the activation of protein kinase A must be altered in chronic epilepsy.

\section{Measurement of the readily releasable pool of glutamate}

So far, the results presented here are compatible with a presynaptic increase in release probability in the kainate model of epilepsy, resulting in a reduced propensity to increase transmitter release in response to various pharmacological and electrophysiological stimulation protocols. It has been experimentally shown in different central synapses that the size of the readily releasable pool of glutamate correlates with the release probability of these synapses (Rosenmund and Stevens, 1996; Dobrunz and Stevens, 1997). Therefore, an increase in the release probability in kainate-treated animals should also lead to an increase in the size of the readily releasable pool. To test this hypothesis, we have compared the size of the readily releasable pool of glutamate in kainate-treated and control animals. For this purpose, we have isolated mossy fiber synaptosomes and induced glutamate release by a short application of hypertonic sucrose (Lonart and Südhof, 1998; Lonart et al., 1998). Basal tritium efflux from large CA3 synaptosomes was stable before sucrose stimulation period $(0.037 \pm 0.0034 / \mathrm{min}$; corresponding to $1.88 \pm 0.17 \mathrm{nCi})$. In synaptosomes from control rats, brief application of sucrose induced a release of $12.49 \pm 0.54 \%$ of ${ }^{3} \mathrm{H}$-glutamate content that was increased by $18.9 \%(n=6 ; p<0.02)$ in kainate-treated rats (Fig. 8A). Similar to intact synapses (Rosenmund and Stevens, 1996), release induced by application of hyperosmolar sucrose was completely independent of $\mathrm{Ca}^{2+}$ influx, and the difference between kainate-treated and control animals persisted after removal of $\mathrm{Ca}^{2+}$ from the extracellular solution (controls, $12.81 \pm$ 


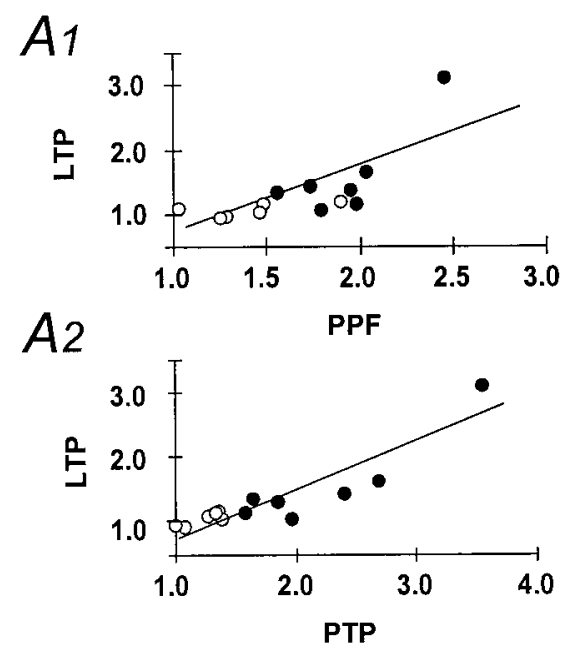

Figure 4. Correlation of facilitation and posttetanic potentiation with LTP. A1, Correlation of paired-pulse facilitation under baseline conditions and the amount of subsequently evoked LTP after $1 \mathrm{hr}$ of posttetanus recording in the mossy fiber pathway. $A 2$, Correlation of posttetanic potentiation and the amount of LTP observed after $1 \mathrm{hr}$. For quantitation of LTP, the fEPSP slope was calculated, and values $1 \mathrm{hr}$ after LTP induction were normalized to the pretetanus baseline. Both correlations were significant at $p<0.05$ (Spearman rank correlation) for control, but not for kainate-treated animals. Open symbols, kainate-treated animals; filled symbols, control animals.

$1.33 \%$; kainate-treated rats, increase by $16.8 \% ; n=5 ; p<0.01$; Fig. 8B).

\section{DISCUSSION}

Synaptic plasticity is an ubiquitous feature of most CNS synapses that permits synapses to retain a trace of previous activity. It has been suggested that synaptic plasticity itself must be subject to regulation to prevent synapses from entering states of saturated LTP or LTD (Abraham and Tate, 1997). In this study, we have asked whether intense neuronal activity induced in vivo by application of kainate leads to long-term changes in short- or longterm plasticity at the mossy fiber synapse. We find that pairedpulse facilitation and augmentation, as well as different forms of LTP, are potently reduced several weeks after kainate-induced status epilepticus. These changes are specific to the mossy fiber pathway and do not affect the associational-commissural input onto CA3 neurons. The common mechanism that underlies these changes may be an increased release probability of the mossy fiber-CA3 synapse.

The release probability of CNS synapses has been suggested to strongly influence both the capacity of CNS synapses to express frequency-dependent short-term modifications as well as LTP. In the CA1 region, synapses showing a low initial probability of release show LTP, whereas synapses with a high initial probability of release do not (Larkman et al., 1992). Similarly, release probability seems to be inversely related to paired-pulse facilitation (Debanne et al., 1996). This suggests that processes that alter the initial setting of the presynaptic release mechanism are important in determining whether short-term plasticity as well as LTP can be expressed. The lasting modification of release probability could be a particularly powerful mechanism for metaplasticity in mossy fiber synapses, in which both short- and long-term synaptic plasticity invoke modifications of neurotransmitter release. For instance, it is thought that paired-pulse facilitation at this synapse is caused by an intraterminal $\mathrm{Ca}^{2+}$ accumulation that enhances

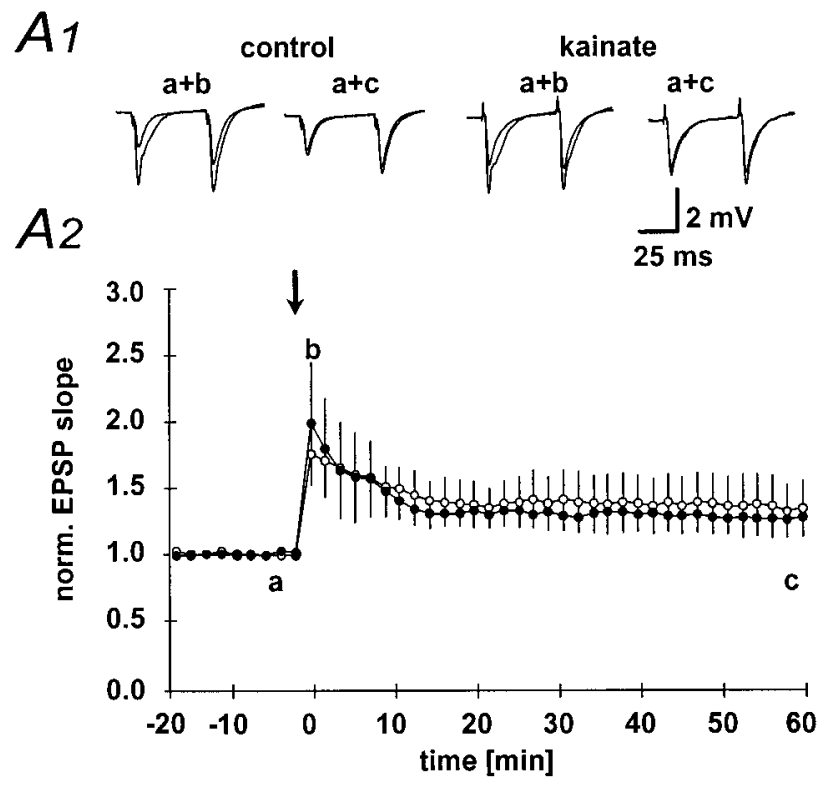

Figure 5. LTP in the associational-commissural pathway. A1, Representative examples of fEPSPs in control and kainate-treated animals collected at the time points indicated by the lowercase letters in A2. A2, Time course of changes in the fEPSP slope after application of a $1 \mathrm{sec}, 100 \mathrm{~Hz}$ tetanus to the associational-commissural pathway. No difference was observed between kainate-treated (open symbols) and control (open symbols) animals.

$\mathrm{Ca}^{2+}$-dependent neurotransmitter release (Regehr et al., 1994). Likewise, the expression of mossy fiber LTP seems to rely primarily on presynaptic processes (Zalutsky and Nicoll, 1990; Nicoll et al., 1994; Xiang et al., 1994; Nicoll and Malenka, 1995).

That neuronal activity during status epilepticus leads to a long-lasting modification of release probability in mossy fiber synapses is suggested by the following key findings. First, pairedpulse facilitation, which is thought to reflect the capacity of mossy fiber-CA3 synapses to increase neurotransmitter release to the second to two closely spaced stimuli is reduced. Concomitantly, the potential to express LTP, which also relies on increased transmitter release, is severely diminished, and the transient decrease in paired-pulse facilitation after LTP induction eliminated. In addition, direct activation of either adenylyl cyclase or protein kinase A caused potentiation in control animals but not in kainate-treated animals. Activation of both enzymes is thought to alter synaptic transmission via a change in neurotransmitter release (Chavez-Noriega and Stevens, 1994; Trudeau et al., 1996), even though activation of postsynaptic protein kinase A may be necessary for full expression of LTP at the mossy fiber synapse (Yeckel et al., 1999). Taken together, these data indicate that status epilepticus results in a reduced propensity to increase mossy fiber transmitter release in response to various pharmacological and electrophysiological stimulation protocols, a finding that would be consistent with a chronically increased neurotransmitter release probability. A direct measurement of the relative size of the readily releasable pool of glutamate (Lonart and Südhof, 1998; Lonart et al., 1998) shows an increase in kainatetreated animals. Because the size of the readily releasable glutamate pool correlates with release probability in hippocampal synapses (Rosenmund and Stevens, 1996; Dobrunz and Stevens, 1997), this result also supports the idea that release probability at the mossy fiber-CA3 synapse is increased. Changes in release 


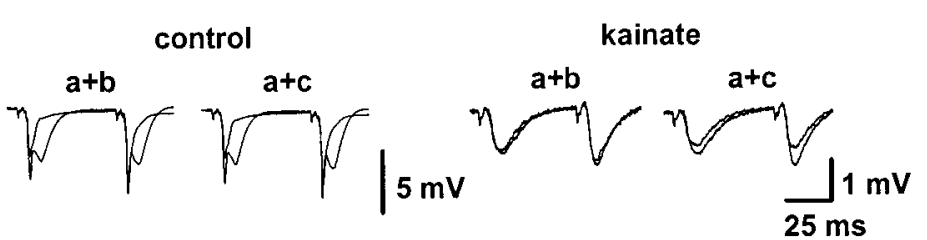

A2

for+IBMX

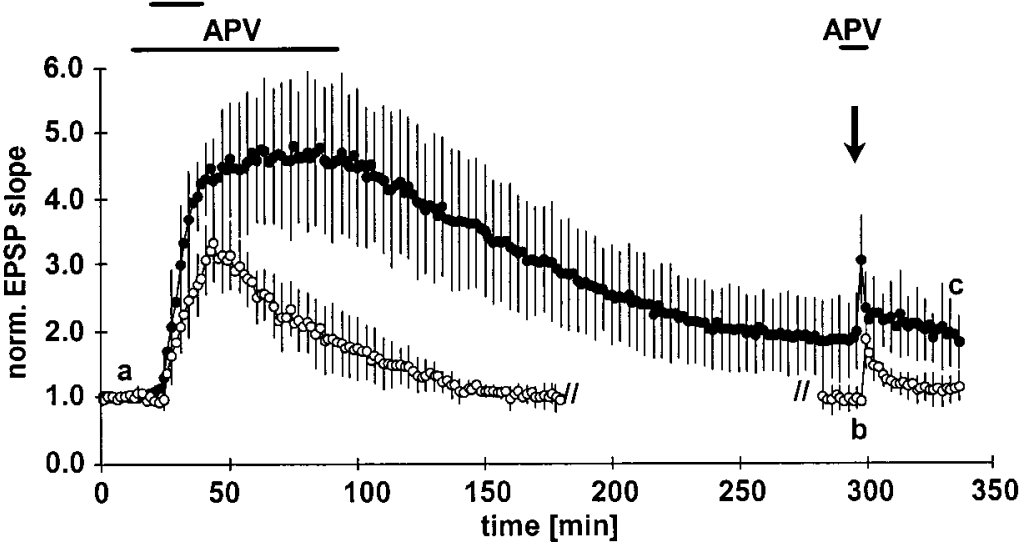

Figure 6. Long-lasting potentiation of mossy fiber fEPSPs by application of forskolin/IBMX. $A 1$, Representative examples of mossy fiber fEPSPs at the time points indicated by the lowercase letters in Figure 5A2. A2, Long-lasting potentiation of fEPSP slope after transient $(20 \mathrm{~min}$, horizontal bar) application of the adenylyl cyclase agonist forskolin (50 $\mu \mathrm{M})$ together with the phosphodiesterase inhibitor IBMX $(50 \mu \mathrm{M})$. fEPSP slope in kainate-treated animals (open symbols) decayed to baseline within $2 \mathrm{hr}$, whereas the fEPSP slope reached stable potentiation after $\sim 4 \mathrm{hr}$. Three hours (kainate-treated animals) or $5.5 \mathrm{hr}$ (control animals, filled symbols) after initiating forskolin/IBMX perfusion, a $100 \mathrm{~Hz}, 1 \mathrm{sec}$ tetanic stimulation was applied to the mossy fiber bundle to demonstrate occlusion of tetanus-induced LTP. For the sake of clarity, the application of a tetanus in kainate-treated animals is displayed at the time point of tetanus application in control animals. No further potentiation could be elicited by tetanization in either group.

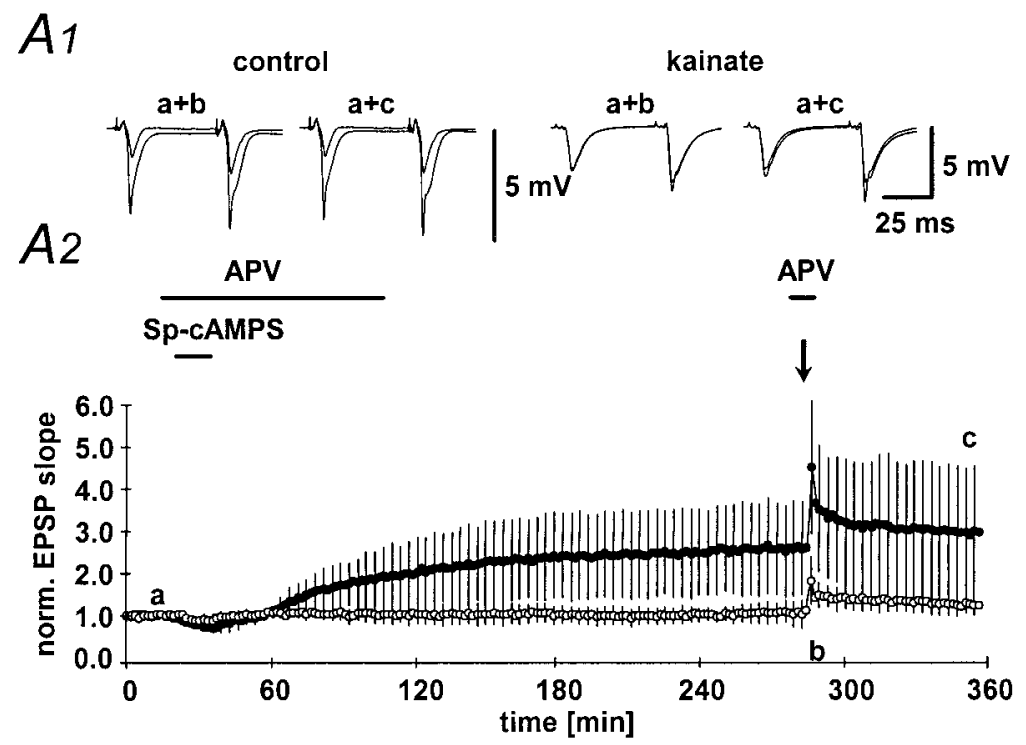

Figure 7. Long-lasting potentiation of mossy fiber fEPSPs by application of Sp-cAMPS. A1, Representative examples of mossy fiber fEPSPs at the time points indicated by the lowercase letters in Figure 6A2. A2, Long-lasting potentiation of fEPSP slope after transient ( $20 \mathrm{~min}$, horizontal bar) application of the cAMP analog Sp-cAMPS $(50 \mu \mathrm{M})$. fEPSP slope in kainate-treated animals (open symbols) and control animals ( filled symbols) was determined. After $4 \mathrm{hr}$, a $100 \mathrm{~Hz}, 1 \mathrm{sec}$ tetanic stimulation was applied to the mossy fiber bundle to demonstrate occlusion of tetanus-induced LTP.

properties after kainate-induced status epilepticus have also been observed for GABAergic synapses in the hippocampal CA1 region. In these synapses, quantal GABA release is deficient (Hirsch et al., 1999). That GABAergic synapses show deficient quantal release, in contrast to increased release in mossy fiber-CA3 synapses and unchanged properties of the associationalcommissural-CA3 synapse, illustrates that plastic changes in the release properties of synapses are highly specific to individual synapse types.

The changes in paired-pulse facilitation could be observed several weeks after kainate-induced status epilepticus, and after a seizure-free period of at least $8 \mathrm{hr}$. This is in marked contrast to changes in paired-pulse facilitation seen after induction of LTP, which are only transient (Regehr and Tank, 1991; Huang et al., 1994). It has been proposed that the expression of late LTP in the mossy fiber-CA3 synapse invokes either (1) the recruitment of novel synapses or active zones with normal paired-pulse facilitation, or (2) stable postsynaptic changes that do not interfere with paired-pulse plasticity (Huang et al., 1994). In this respect, the kainate-induced change in presynaptic function is distinct from the induction of LTP.

Interestingly, mutant mice lacking the $\mathrm{Ca}^{2+}$-binding protein calbindin- $\mathrm{D}_{28 \mathrm{k}}$ in presynaptic mossy fiber terminals also show reduced facilitation and frequency potentiation in the mossy fiber-CA3 synapse (Klapstein et al., 1998). This $\mathrm{Ca}^{2+}$-binding protein is also lost in the kainate model of epilepsy (Yang et al., 1997). The evidence from mutant mice suggests that chronically impaired presynaptic $\mathrm{Ca}^{2+}$ homeostasis within mossy fiber terminals may be sufficient to lead to a long-term change in release probability. Alternatively, the altered properties of mossy fiber to CA3 synaptic transmission may be related to synaptic sprouting of mossy fibers in kainate-treated animals. In these animals, novel 


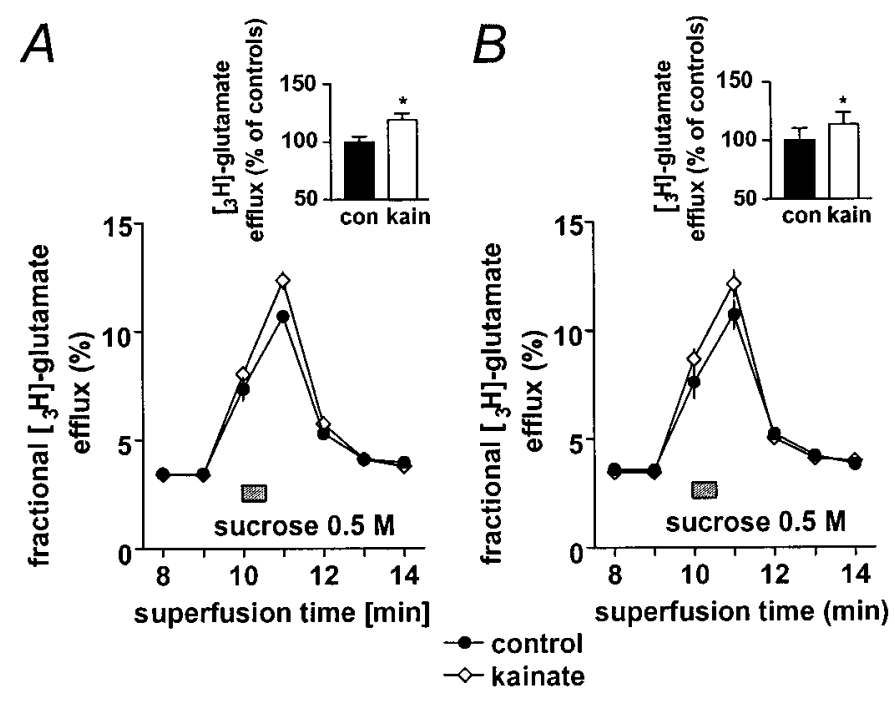

Figure 8. Measurement of the readily releasable pool of glutamate in mossy fiber synaptosomes. $A, B,{ }^{3} \mathrm{H}$-Glutamate release from rat $\mathrm{CA} 3$ mossy fiber synaptosomes induced by addition of $0.5 \mathrm{M}$ sucrose (horizontal bars). Synaptosomes superfused with $\mathrm{Ca}^{2+}$-containing (Fig. 8A) or $\mathrm{Ca}^{2+}$-free (Fig. $8 B$ ) Krebs' buffer were exposed for $30 \mathrm{sec}$ to $0.5 \mathrm{M}$ sucrose (horizontal bars). ${ }^{3} \mathrm{H}$-Glutamate release traces represent percentage of the total ${ }^{3} \mathrm{H}$-glutamate present in the synaptosomes at the beginning of the respective collection period. Inserts show the area-under-the-curve of the respective traces in percentages of the controls (con) without kainate treatment.

synaptic contacts are formed by aberrant mossy fiber collaterals onto CA3 neurons and the proximal dendrites of dentate granule neurons. These novel synapses might conceivably show decreased short- and long-term plasticity.

Irrespective of the reasons for the change in mossy fiber synaptic transmission, we suggest that altered release probability may profoundly affect the input-output properties of the CA3 region. In the cortex, altered release probability of synapses between cortical neurons results in a redistribution of synaptic efficacy during individual synaptic events in a train (Tsodyks and Markram, 1997). According to these authors, one consequence of saturated release probability would be that the extent to which rate coding is possible would be diminished. In addition, synaptic connections showing a low release probability with a high degree of paired-pulse facilitation are highly effective in transmitting high-frequency bursts (Lisman, 1997), a property that would be considerably altered in mossy fiber-CA3 synapses after status epilepticus. It remains to be seen whether more physiological forms of neuronal activity can also cause changes of release probability similar to those seen here. However, these data indicate that lasting changes in neurotransmitter release probability caused by neuronal activity may be a powerful mechanism for metaplasticity that modulates both short- and long-term plasticity in the mossy fiber-CA3 synapse.

\section{REFERENCES}

Abel T, Nguyen PV, Barad M, Deuel TA, Kandel ER, Bourtchouladze R (1997) Genetic demonstration of a role for PKA in the late phase of LTP and in hippocampus-based long-term memory. Cell 88:615-626.

Abraham WC, Tate WP (1997) Metaplasticity: a new vista across the field of synaptic plasticity. Prog Neurobiol 52:303-323.

Bortolotto ZA, Bashir ZI, Davies CH, Collingridge GL (1994) A molecular switch activated by metabotropic glutamate receptors regulates induction of long-term potentiation. Nature 368:740-743.
Castillo PE, Salin PA, Weisskopf MG, Nicoll RA (1996) Characterizing the site and mode of action of dynorphin at hippocampal mossy fiber synapses in the guinea pig. J Neurosci 16:5942-5950.

Castillo PE, Janz R, Sudhof TC, Tzounopoulos T, Malenka RC, Nicoll RA (1997) Rab3A is essential for mossy fibre long-term potentiation in the hippocampus. Nature 388:590-593.

Chavez-Noriega LE, Stevens CF (1994) Increased transmitter release at excitatory synapses produced by direct activation of adenylate cyclase in rat hippocampal slices. J Neurosci 14:310-317.

Claiborne BJ, Xiang Z, Brown TH (1993) Hippocampal circuitry complicates analysis of long-term potentiation in mossy fiber synapses. Hippocampus 3:115-121.

Cohen AS, Raymond CR, Abraham WC (1998) Priming of long-term potentiation induced by activation of metabotropic glutamate receptors coupled to phospholipase C. Hippocampus 8:160-170.

Debanne D, Guerineau NC, Gahwiler BH, Thompson SM (1996) Paired-pulse facilitation and depression at unitary synapses in rat hippocampus: quantal fluctuation affects subsequent release. J Physiol (Lond) 491:163-176.

Dobrunz LE, Stevens CF (1997) Heterogeneity of release probability, facilitation, and depletion at central synapses. Neuron 18:995-1008.

Fink K, Göthert M (1992) Presynaptic site of action underlying the ethanol-induced inhibition of norepinephrine release evoked by stimulation of $\mathrm{N}$-methyl-D-aspartate (NMDA) receptors in rat cerebral cortex. Brain Res 572:27-32.

Frey U, Huang YY, Kandel ER (1993) Effects of cAMP simulate a late stage of LTP in hippocampal CA1 neurons. Science 260:1661-1664.

Hirsch JC, Agassandian C, Merchan-Perez A, Ben Ari Y, DeFelipe J, Esclapez M, Bernard C (1999) Deficit of quantal release of GABA in experimental models of temporal lobe epilepsy [see comments]. Nat Neurosci 2:499-500.

Hortnagl H, Berger ML, Sperk G, Pifl C (1991) Regional heterogeneity in the distribution of neurotransmitter markers in the rat hippocampus. Neuroscience 45:261-272.

Huang YY, Li XC, Kandel ER (1994) cAMP contributes to mossy fiber LTP by initiating both a covalently mediated early phase and macromolecular synthesis-dependent late phase. Cell 79:69-79.

Huang YY, Kandel ER, Varshavsky L, Brandon EP, Qi M, Idzerda RL, McKnight GS, Bourtchouladze R (1995) A genetic test of the effects of mutations in PKA on mossy fiber LTP and its relation to spatial and contextual learning. Cell 83:1211-1222.

Klapstein GJ, Vietla S, Lieberman DN, Gray PA, Airaksinen MS, Thoenen H, Meyer M, Mody I (1998) Calbindin- $\mathrm{D}_{28 \mathrm{~K}}$ fails to protect hippocampal neurons against ischema in spite of its cytoplasmic calcium buffering properties: evidence from calbindin- $\mathrm{D}_{28 \mathrm{~K}}$ knockout mice. Neuroscience 85:361-373.

Larkman A, Hannay T, Stratford K, Jack J (1992) Presynaptic release probability influences the locus of long term potentiation. Nature 360:70-72.

Lisman JE (1997) Bursts as a unit of neural information: making unreliable synapses reliable [see comments]. Trends Neurosci 20:38-43.

Lonart G, Südhof TC (1998) Region-specific phosphorylation of rabphilin in mossy fiber nerve terminals of the hippocampus. J Neurosci 18:634-640.

Lonart G, Janz R, Johnson KM, Südhof TC (1998) Mechanism of action of rab3A in mossy fiber LTP. Neuron 21:1141-1150.

Nicoll RA, Malenka RC (1995) Contrasting properties of two forms of long-term potentiation in the hippocampus. Nature 377:115-118.

Nicoll RA, Castillo PE, Weisskopf MG (1994) The role of $\mathrm{Ca}^{2+}$ in transmitter release and long-term potentiation at hippocampal mossy fiber synapses. Adv Second Messenger Phosphoprotein Res 29:497-505.

Racine RJ (1972) Modification of seizure activity by electrical stimulation: I. After-discharge threshold. Electroencephalogr Clin Neurophysiol 32:269-279.

Regehr WG, Tank DW (1991) The maintenance of LTP at hippocampal mossy fiber synapses is independent of sustained presynaptic calcium. Neuron 7:451-459.

Regehr WG, Delaney KR, Tank DW (1994) The role of presynaptic calcium in short-term enhancement at the hippocampal mossy fiber synapse. J Neurosci 14:523-537.

Rosenmund C, Stevens CF (1996) Definition of the readily releasable pool of vesicles at hippocampal synapses. Neuron 16:1197-1207.

Salin PA, Scanziani M, Malenka RC, Nicoll RA (1996) Distinct short- 
term plasticity at two excitatory synapses in the hippocampus. Proc Natl Acad Sci USA 93:13304-13309.

Trudeau LE, Emery DG, Haydon PG (1996) Direct modulation of the secretory machinery underlies PKA-dependent synaptic facilitation in hippocampal neurons. Neuron 17:789-797.

Tsodyks MV, Markram H (1997) The neural code between neocortical pyramidal neurons depends on neurotransmitter release probability [Erratum (1997) 94:5495] Proc Natl Acad Sci USA 94:719-723.

Villacres EC, Wong ST, Chavkin C, Storm DR (1998) Type I adenylyl cyclase mutant mice have impaired mossy fiber long-term potentiation. J Neurosci 18:3186-3194.

Wang H, Wagner JJ (1999) Priming-induced shift in synaptic plasticity in the rat hippocampus. J Neurophysiol 82:2024-2028.

Weisskopf MG, Castillo PE, Zalutsky RA, Nicoll RA4 (1994) Media- tion of hippocampal mossy fiber long-term potentiation by cyclic amp. Science 265:1878-1882.

Xiang Z, Greenwood AC, Kairiss EW, Brown TH (1994) Quantal mechanism of long-term potentiation in hippocampal mossy-fiber synapses. J Neurophysiol 71:2552-2556.

Yang Q, Wang S, Hamberger A, Celio MR, Haglid KG (1997) Delayed decrease of calbindin immunoreactivity in the granule cell- mossy fibers after kainic acid-induced seizures. Brain Res Bull 43:551-559.

Yeckel MF, Kapur A, Johnston D (1999) Multiple forms of LTP in hippocampal CA3 neurons use a common postsynaptic mechanism. Nat Neurosci 2:625-633.

Zalutsky RA, Nicoll RA (1990) Comparison of two forms of long-term potentiation in single hippocampal neurons [Erratum (1991) 251:856]. Science 248:1619-1624. 